\title{
REDUCIBLE VERONESE SURFACES
}

\author{
ALBERTO ALZATI AND EDOARDO BALLICO
}

\begin{abstract}
Here we describe all degree $n+3$ non-degenerate surfaces in $\mathbb{P}^{n+4}$, $n \geq 1$, connected in codimension 1 , which may be isomorphically projected into $\mathbb{P}^{4}$. There are 3 of them. One is a suitable union of $n+3$ planes (for all $n \geq 1$ ); it was discovered by Floystad. The other two are unions of a smooth quadric and two planes (only for $n=1$ ).
\end{abstract}

\section{INTRODUCTION}

Let $\mathbb{P}^{N}$ be the $N$-dimensional projective space on $\mathbb{C}$. For any integer $k \geq 0$, a reduced subvariety $V \subset \mathbb{P}^{N}$ of pure dimension is said to be connected in codimension $k$ if for any closed subvariety $W \subset V$, such that $\operatorname{cod}_{V}(W)>k$, we have that $V \backslash W$ is connected. For any subvariety $V \subset \mathbb{P}^{N}$ and for any $\lambda$-dimensional linear subspace $\Lambda \subset \mathbb{P}^{N}$ we say that $V$ projects isomorphically to $\Lambda$ is there exists a linear projection $\pi_{\mathcal{L}}: \mathbb{P}^{N}-->\Lambda$, from a suitable $(N-\lambda-1)$-dimensional linear space $\mathcal{L}$, disjoint from $V$, such that $\pi_{\mathcal{L}}(V)$ is isomorphic to $V$.

In this note we consider the following type of surface arising from the example decribed in $\S 2$.

Definition 1. For any positive integer $n \geq 1$, we will call reducible Veronese surface any algebraic surface $X \subset \mathbb{P}^{n+4}$ such that:

i) $X$ is a non degenerated, reduced, reducible surface of pure dimension 2;

ii) $\operatorname{deg}(X)=n+3, \operatorname{cod}(X)=n+2$, so that $X$ is a minimal degree surface;

iii) $\operatorname{dim}[\operatorname{Sec}(X)] \leq 4$, so that it is possible to choose a generic linear space $\mathcal{L}$ of dimension $n-1$ in $\mathbb{P}^{n+4}$ such $\pi_{\mathcal{L}}(X)$ is isomorphic to $X$, where $\pi_{\mathcal{L}}$ is the the rational projection $\pi_{\mathcal{L}}: \mathbb{P}^{n+4}--->\Lambda$, from $\mathcal{L}$ to a generic target $\Lambda \simeq \mathbb{P}^{4}$

iv) $X$ is connected in codimension 1 , i.e. if we drop any finite number (eventually $0)$ of points $Q_{1}, \ldots, Q_{r}$ from $X$ we have that $X \backslash\left\{Q_{1}, \ldots, Q_{r}\right\}$ is connected;

v) $X$ is a locally Cohen-Macaulay surface.

Remark 1. Actually v) implies iv) by cor. 2.4 of $[\mathrm{H}]$, however we think that it is more useful to give the above definition 1 because condition iv) is crucial to get the classification.

In summary: we prove that there are exactly 3 types of reducible Veronese surfaces:

Date: March 9, 2009.

1991 Mathematics Subject Classification. Primary 14J25; Secondary 14N20.

Key words and phrases. reducible surfaces, projectability.

This work is within the framework of the national research projects: "Geometry on Algebraic Varieties" Cofin 2006 of MIUR and "Geometric Properties of Real and Complex Varieties" Cofin 2007 of MIUR. 
(i) a suitable union of $n+3$ planes (for any integr $n \geq 1$ ) which sits as a linearly normal scheme in $\mathbb{P}^{n+4}$ (see theorem 2 and definition 2 for a precise description); these are the examples whose existence is proved in $[\mathrm{F}]$;

(ii) two surfaces which are union of a smooth quadric surface and two planes; each of these two examples sits as a linearly normal scheme in $\mathbb{P}^{5}$ (see theorems 3 for their description);

and there are no other cases (see proposition 2 and theorem 4).

We will use the following definitions:

$<V_{1} \cup \ldots \cup V_{r}>$ : linear span in $\mathbb{P}^{N}$ of the subvarieties $V_{i} \subset \mathbb{P}^{N}, i=1, \ldots, r$;

$\operatorname{Supp}(V)$ : support of the subscheme $V \subset \mathbb{P}^{N}$;

$\operatorname{Sing}(V)$ : singular locus of the subscheme $V \subset \mathbb{P}^{N}$;

$\operatorname{Sec}(V): \overline{\left\{\bigcup_{v_{1} \neq v_{2} \in V}<v_{1} \cup v_{2}>\right\}} \subset \mathbb{P}^{N}$ for any subvariety $V \subset \mathbb{P}^{N}$.

For any positive integer $d \geq 2$ a rational comb of degree $d$ in $\mathbb{P}^{N}$ is the union of $d$ lines $L_{1}, L_{2}, \ldots, L_{d} \subset \mathbb{P}^{N}$ such that, for any $i \geq 2, L_{i} \cap L_{1}$ is a point, these $d-1$ points are distinct and, for any $j>i \geq 2, L_{i} \cap L_{j}=\emptyset$.

\section{Floystad's EXAMPLE}

In $[\mathrm{F}]$, corollary 3 , the author proves that, for any integer $n \geq 1$, there exists in $\mathbb{P}^{4}$ a monad of the following form:

$$
\mathcal{O}_{\mathbb{P}^{4}}(-1)^{\oplus n+2} \rightarrow \mathcal{O}_{\mathbb{P}^{4}}^{\oplus 2 n+3} \rightarrow \mathcal{O}_{\mathbb{P}^{4}}(1)^{\oplus n}
$$

whose homology is $\mathcal{I}_{S_{n}}(2)$ where $S_{n}$ is a locally Cohen-Macaulay surface in $\mathbb{P}^{4}$. Moreover $S_{n}$ is embedded in $\mathbb{P}^{n+4}$ as a linearly normal surface and $S_{n}$ projects isomorphically to some suitable $\Lambda \subset \mathbb{P}^{n+4}, \Lambda \simeq \mathbb{P}^{4}$. For $n=1, S_{1}$ is the usual (smooth) Veronese surface in $\mathbb{P}^{5}$; on the contrary, $S_{n}$ must be singular for $n \geq 2$.

If we call $\varphi_{n}: \mathcal{O}_{\mathbb{P}^{4}}{ }^{\oplus 2 n+3} \rightarrow \mathcal{O}_{\mathbb{P}^{4}}(1)^{\oplus n}$ we get the following exact sequences of sheaves and vector bundles over $\mathbb{P}^{4}$ :

$$
\begin{gathered}
0 \rightarrow \operatorname{ker}\left(\varphi_{n}\right) \rightarrow \mathcal{O}_{\mathbb{P}^{4}}{ }^{\oplus 2 n+3} \rightarrow \mathcal{O}_{\mathbb{P}^{4}}(1)^{\oplus n} \rightarrow 0 \\
0 \rightarrow \mathcal{O}_{\mathbb{P}^{4}}(-1)^{\oplus n+2} \rightarrow \operatorname{ker}\left(\varphi_{n}\right) \rightarrow \mathcal{I}_{S_{n}}(2) \rightarrow 0 .
\end{gathered}
$$

Now it is easy to calculate $\chi\left[\mathcal{O}_{S_{n}}(t)\right]=\left(\begin{array}{c}t+4 \\ 4\end{array}\right)-\chi\left[\mathcal{I}_{S_{n}}(t)\right]=\left(\frac{n+3}{2}\right) t^{2}+\left(\frac{n+5}{2}\right) t+1$, so that $\operatorname{deg}\left(S_{n}\right)=n+3$ and $S_{n}$ is a minimal degree surface in $\mathbb{P}^{n+4}$ for any $n \geq 1$.

When $n=2$, by a computer algebra system as Macaulay, it is easy to get a set of generators for the ideal of a generic $S_{2}$ in $\mathbb{P}^{6}$. In fact, by choosing a random $(2,7)$ matrix $M$ of linear forms we have a map as $\varphi_{n}$ and, by calculating the higher sizygies of $M$, we get a free resolution for $\operatorname{ker}\left(\varphi_{n}\right)$ and a commutative diagram as follows:

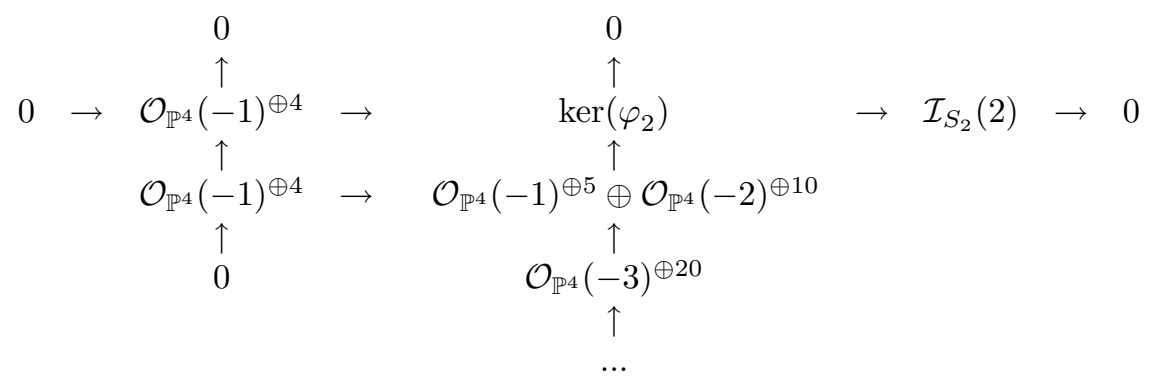

By choosing another random $(5,4)$ matrix $N$ of constants, in order to get a map $\mathcal{O}_{\mathbb{P}^{4}}(-1)^{\oplus 4} \rightarrow \mathcal{O}_{\mathbb{P}^{4}}(-1)^{\oplus 5},\left(\mathcal{O}_{\mathbb{P}^{4}}(-1)^{\oplus 4} \rightarrow \mathcal{O}_{\mathbb{P}^{4}}(-2)^{\oplus 10}\right.$ is the zero map) and by 
using the mapping cone technique, we have that the ideal $I_{S_{2}}$ in $\mathbb{P}^{6}$ of a generic surface $S_{2}$ is generated by one cubic and ten quartics. $S_{2}$ has codimension 4 , degree 5 and (arithmetic) sectional genus 0. Alternatively, one can also choose 4 generic sections of the rank 5 vector bundle $\operatorname{ker}\left(\varphi_{2}\right) \otimes \mathcal{O}_{\mathbb{P}^{4}}(1)$ by giving a random $(5,4)$ matrix of constants $N^{\prime}$ : in this case $S_{2}$ is the degeneracy locus in $\mathbb{P}^{6}$ of these sections; if $N^{\prime}=N$ we get exactly the same set of generators for $I_{S_{2}}$.

By knowing a set of generators for $I_{S_{2}}$ it is, more or less, easy to see that the generic $S_{2}$ is given by 5 planes $\Pi_{0}, \Pi_{1}, \ldots, \Pi_{4}$ such that: $\Pi_{0} \cap \Pi_{i}:=L_{i}$ is a line for $i=1, \ldots, 4 ; \Pi_{i} \cap \Pi_{j}:=Q_{i j}$ is a point of $\Pi_{0}$ for $i, j=1, \ldots, 4, i \neq j$, and the lines $L_{i}$ are in general position on $\Pi_{0}$. The generic hyperplane section of $S_{2}$ is a rational comb of degree 5 given by a line $l_{0}$ on $\Pi_{0}$ and four other lines $l_{i}, i=1, \ldots, 4, l_{i} \in \Pi_{i}$, $l_{i} \cap l_{j}=\emptyset$ for $i \neq j$, intersecting $l_{0}$ at one point. $\operatorname{Sec}\left(S_{2}\right)$ is the union of a finite number of linear spaces of dimension $2\left(\Pi_{i} i=0, \ldots, 4\right), 3\left(<\Pi_{0} \cup \Pi_{i}>, i=1, \ldots, 4\right)$ or $4\left(<\Pi_{i} \cup \Pi_{j}>, i, j=1, \ldots, 4, i \neq j\right)$ so that it is possible to choose a generic line $\mathcal{L}$ in $\mathbb{P}^{6}, \mathcal{L} \cap \operatorname{Sec}\left(S_{2}\right)=\emptyset$, and to project $S_{2}$, from $\mathcal{L}$ to a generic $\Lambda \simeq \mathbb{P}^{4}$, in such a way that the projection of $S_{2}$ is isomorphic to $S_{2}$.

The above concrete construction of $S_{2}$ suggests to define a family of completely reducible surfaces having the same properties.

Definition 2. For any positive integer $n \geq 1$, let us choose a plane $\Pi_{0}$ and $n+2$ distinct points $P_{1}, \ldots, P_{n+2}$ in general position in $\mathbb{P}^{n+4}$, so that $<\Pi_{0} \cup P_{1} \cup \ldots \cup$ $P_{n+2}>=\mathbb{P}^{n+4}$. Let us choose $n+2$ planes $\Pi_{i}, i=1, \ldots, n+2, P_{i} \in \Pi_{i}$, such that $\Pi_{i} \cap \Pi_{0}$ is a line $L_{i}$ and the $n+2$ lines $L_{i}$ are in general position on $\Pi_{0}$. (i.e. that the curve given by their union has no triple points). Let us call $\Sigma_{n}$ any surface in $\mathbb{P}^{n+4}$ which is the union $\Pi_{0} \cup \Pi_{1} \ldots \cup \Pi_{n+2}$.

Proposition 1. The previously defined surfaces $\Sigma_{n}, n \geq 1$, are reducible Veronese surfaces according to definition 1.

Proof. $i), i i), i i i), i v)$ follow directly from the definition, note that $\operatorname{Sec}\left(\Sigma_{n}\right)$ is the union of a finite number of linear spaces of dimension 2,3,4. As far concerning $v$ ), let us remark that for any singular point $P \in \Sigma_{n}$ its local ring is isomorphic either:

- to the local ring at $(0,0,0)$ of the affine variety $\{x y=0\}$ in $\mathbb{A}^{3}(\mathbb{C})$

or:

- to the local ring at $(0,0,0,0)$ of the affine variety

$\{x=y=0\} \cup\{z=w=0\} \cup\{x=z=0\}=$

$=\left\{x^{2} z=x z^{2}=x^{2} w=x z w=x y z=y z^{2}=x y w=y z w=0\right\}$ in $\mathbb{A}^{4}(\mathbb{C})$.

They are, up to isomorphisms, the same local rings of the singular points of $S_{2}$ and we know that $S_{2}$ is a locally Cohen-Macaulay surface by cor. 3 of $[\mathrm{F}]$.

To prove that $\Sigma_{n}$ are locally Cohen-Macaulay we could also use a slightly different version of the following lemma which will be useful at the end of the paper.

Lemma 1. Let $X \subset \mathbb{P}^{5}$ be a non degenerate surface such that $X=Q \cup X_{1} \cup X_{2}$, where $Q$ is a smooth quadric, $X_{1}$ and $X_{2}$ are planes, and either $X_{1}$ and $X_{2}$ cut $Q$ along two lines intersecting at a point $P=X_{1} \cap X_{2}$ or $Q, X_{1}, X_{2}$ intersect transversally along a unique line $L=Q \cap X_{1} \cap X_{2}$. Then $X$ is a locally CohenMacaulay surface.

Proof. Let us consider the first case. Obviously we have to check the property only at $P$. Let $R$ be the local ring of $X$ at $P$ and let $m$ be its maximal ideal. We have $h e i g h t(m)=2$, so that we have to prove that $\operatorname{depth}(m)=2$. As $X$ is reduced and 
$\operatorname{dim}(X) \geq 1$ we know that $\operatorname{depth}(m) \geq 1$. A generic hyperplane section of $X$ not passing through $P$ cuts $X$ along a reducible curve $Y=C \cup L_{1} \cup L_{2}$, where $C$ is a smooth conic and $L_{1}, L_{2}$ are two disjoint lines intersecting $C$ transversally at two different points. $Y$ is reduced, connected and its arithmetic genus $p_{a}(Y)$ is 0 . Let $H$ be a generic hyperplane section of $X$ passing through $P$, now $H \cap X:=Y_{P}$ is reducible as the union of a smooth conic $C_{P}$ and two distinct lines intersecting $C_{P}$ transversally at $P$. $H$ gives rise to a non zero divisor element $\alpha \in m$ because $X$ has pure dimension 2 . Now let us remark that $p_{a}\left(Y_{P}\right)=0$, so that $Y_{P}$ has no embedded components at $P=\operatorname{Sing}\left(Y_{P}\right)$, otherwise $p_{a}\left(Y_{P}\right)<p_{a}(Y)$. Hence there is at least a non zero divisor element $\beta \in m /(\alpha)$ and $(\alpha, \beta)$ is a regular sequence for $m$, so that $\operatorname{depth}(m) \geq 2$. As $\operatorname{depth}(m) \leq \operatorname{height}(m)=2$ we are done.

In the second case we can argue as in the previous one for all points $P \in L$.

Remark 2. It is easy to see that the generic section of $\Sigma_{n}$ is a rational comb, quite exactly as in the case of $S_{2}$ (which is in fact an example of $\Sigma_{2}$ ), so that $p_{a}\left(\Sigma_{n}\right)=0$, but we will not consider this property in the sequel.

Now it is very natural to ask if the surfaces $\Sigma_{n}$ are the only existing reducible Veronese surfaces in our sense. The answer to this question is the aim of the following sections. Moreover we will prove that any generic $S_{n}$ is a surface $\Sigma_{n}$ for $n \geq 2$, see Remark 3 . To show that the matter is in fact very intricate, let us consider the following:

Example 1. Let $X=Q \cup \Pi_{1} \cup \Pi_{2} \cup \Pi_{3} \subset \mathbb{P}^{6}$, where $Q$ is a smooth quadric of $\mathbb{P}^{3}$ and any $\Pi_{i}$ is a generic plane such that, if we call the three points $P_{i j}:=\Pi_{i} \cap \Pi_{j}$, we have: $P_{i j} \notin \Pi_{k}$ for $k \neq i, j, P_{i j} \notin Q$, but $P_{i j} \in<Q>$. Then $X$ is non degenerated, $\operatorname{deg}(X)=5, \operatorname{dim}[\operatorname{Sec}(X)] \leq 4$, but $X$ is not connected in codimension 1 , for instance because $X \backslash\left\{P_{12} \cup P_{23} \cup P_{31}\right\}$ is not connected.

\section{XAmbÒ's RESUlt AND APPLiCATiOns}

In $[\mathrm{X}]$ Xambò proves the following result:

Theorem 1. Let $V=V_{1} \cup \ldots \cup V_{r} \subset \mathbb{P}^{N}$ be a non degenerate, reducible, reduced, surface of pure dimension 2 , whose irreducible components are $V_{1}, \ldots, V_{r}$. Assume that $V$ is connected in codimension 1 and that it has minimal degree, then:

- any irreducible component $V_{i}$ of dimension 2 of $V$ is a surface of minimal degree in its span $<V_{i}>$;

- there is at least an ordering $V_{1}, V_{2}, \ldots V_{r}$ such that, for any $j=2, \ldots, r$, $V_{j} \cap\left(V_{1} \cup \ldots \cup V_{j-1}\right)=\left\langle V_{j}>\cap<V_{1} \cup \ldots \cup V_{j-1}>\right.$ and this intersection is always a line.

Proof. The theorem is a simply consequence of th. 1 of $[\mathrm{X}]$.

Corollary 1. Let $\Pi_{1}, \Pi_{2}, \ldots, \Pi_{r}$ be a set of ordered planes in some $\mathbb{P}^{N}$ such that:

i) $<\Pi_{1} \cup \Pi_{2} \cup \ldots \cup \Pi_{r}>=\mathbb{P}^{N}$;

ii) for any $j \geq 2, \operatorname{dim}\left(\Pi_{j} \cap<\Pi_{1} \cup \ldots \cup \Pi_{j-1}>\right)=1$;

then $X:=\Pi_{1} \cup \Pi_{2} \cup \ldots \cup \Pi_{r}$ is a non degenerated surface in $\mathbb{P}^{N}$, of minimal degree, connected in codimension 1.

Proof. The lemma follows from the Remark after th. 1 of $[\mathrm{X}]$, p. 151. 
Corollary 2. Let $V$ be any surface as in theorem 1, then for any pair of irreducible components $V_{j}, V_{k} \subset V$ we have only three possibilities:

$-V_{j} \cap V_{k}=\emptyset$

- $V_{j} \cap V_{k}$ is a point

- $V_{j} \cap V_{k}$ is a line.

Proof. Let us assume that $V_{j} \cap V_{k} \neq \emptyset$ and that $k>j$ in the existing ordering of the components of $V$ considered by theorem 1. Then $V_{j} \cap V_{k} \subseteq V_{k} \cap\left(V_{1} \cup \ldots V_{j} \cup \ldots \cup V_{k-1}\right)$ which is a line, as a scheme, because it is the intersection of two linear spaces in $\mathbb{P}^{N}$. By th. 0.4 of [E-G-H-P] $V$ is small according to the definition of [E-G-H-P], p.1364, hence $V_{j} \cap V_{k}=\left\langle V_{i}\right\rangle \cap\left\langle V_{j}\right\rangle$ is a linear space by prop. 2.4 of [E-G-H-P]. As $V_{j} \cap V_{k}$ is contained in a line corollary 2 follows.

Lemma 2. Let $X \subset \mathbb{P}^{n+4}$ be a reducible Veronese surface, according to definition 1 , for some $n \geq 1$. Then:

i) any connected surface $Y \subset X$ can be isomorphically projected in $\mathbb{P}^{4}$;

ii) for any pair of irreducible components $X_{j}$ and $X_{k}$ of $X$ we have $X_{j} \cap X_{k} \neq \emptyset$.

Proof. As $X$ is a reducible Veronese surface there exists a projection $\pi_{\mathcal{L}}: \mathbb{P}^{n+4}-$ $-->\Lambda$, from a suitable linear space $\mathcal{L}$ to a suitable linear space $\Lambda \subset \mathbb{P}^{n+4}, \Lambda \simeq$ $\mathbb{P}^{4}$, such that $\pi_{\mathcal{L}}(X) \simeq X$. This implies that, for any $i=1, \ldots, r, \pi_{\mathcal{L}}\left(X_{i}\right) \simeq X_{i}$, and, for any pair $X_{j}, X_{k} \subset X, \pi_{\mathcal{L}}\left(X_{j}\right) \cap \pi_{\mathcal{L}}\left(X_{k}\right) \simeq X_{j} \cap X_{k}$. Hence for any surface $Y \subset X$ we have $\pi_{\mathcal{L}}(Y) \simeq Y$ and $\pi_{\mathcal{L}}\left(X_{j}\right) \cap \pi_{\mathcal{L}}\left(X_{k}\right)$, being the intersection of two surfaces in $\mathbb{P}^{4}$, can not be empty, so that $X_{j} \cap X_{k}$ can not be empty too.

Lemma 3. Let $X \subset \mathbb{P}^{n+4}$ be a reducible Veronese surface, according to definition 1, for some $n \geq 1$. Let $P$ be a singular point of $X$ and let $X_{1}^{P}, \ldots, X_{s}^{P}$ be the irreducible components of $X$ containing $P$ with $s \geq 2$. For any $i=1, \ldots, s$ let $T_{i}$ be the tangent space of $X_{i}^{P}$ at $P$ in $\left\langle X_{i}^{P}\right\rangle$ and let us assume that the natural ordering of $X_{1}^{P}, \ldots, X_{s}^{P}$ is coherent with the ordering given by theorem 1. Then, for any $j \geq 2, T_{j} \nsubseteq<T_{1} \cup \ldots \cup T_{j-1}>$ and $\operatorname{dim}\left[T_{j} \cap<T_{1} \cup \ldots \cup T_{j-1}>\right] \leq 1$.

Proof. By contradiction, let us assume that $T_{j} \subseteq<T_{1} \cup \ldots \cup T_{j-1}>$, hence $T_{j} \subseteq$ $T_{j} \cap<T_{1} \cup \ldots \cup T_{j-1}>\subseteq<X_{j}^{P}>\cap<X_{1}^{P} \cup \ldots \cup X_{j-1}^{P}>$. As we are assuming that the natural ordering of $X_{1}^{P}, \ldots, X_{s}^{P}$ is coherent with the ordering given by theorem 1, we have that $\operatorname{dim}\left[<X_{j}^{P}>\cap<X_{1}^{P} \cup \ldots \cup X_{j-1}^{P}>\right] \leq 1$. Moreover $\operatorname{dim}\left(T_{j}\right)=2$ if $P$ is a smooth point of $X_{j}^{P}$ and $\operatorname{dim}\left(T_{j}\right)=3$ if $P$ is a singular point of $X_{j}^{P}$; in fact by theorem 1 we know that every $X_{j}$ is an irreducible, reduced, surface of minimal degree in its span and from the well known classification of these surfaces (see for instance th. 0.1 of $[\mathrm{E}-\mathrm{G}-\mathrm{H}-\mathrm{P}]$ ) we have that $X_{j}$ is singular if and only if it is a rank 3 quadric. So that in any case we get a contradiction. By the way we have also proved that $\operatorname{dim}\left[T_{j} \cap<T_{1} \cup \ldots \cup T_{j-1}>\right] \leq 1$.

Lemma 4. Let $X \subset \mathbb{P}^{n+4}$ be a reducible Veronese surface, according to definition 1 , for some $n \geq 1$. Let $P$ be any point of $X$ and let $X_{1}^{P}, \ldots, X_{s}^{P}$ be the irreducible components of $X$ containing $P, s \geq 1$. For any $i=1, \ldots, s$ let $T_{i}$ be the tangent space of $X_{i}^{P}$ at $P$ in $\left\langle X_{i}^{P}>\right.$ and let $\mathbb{T}_{P}:=\bigcup_{i=1}^{s} T_{i}$. Then $\operatorname{dim}\left(<\mathbb{T}_{P}>\right) \leq 4$.

Proof. If $s=1$ we have that $\left\langle\mathbb{T}_{P}\right\rangle=T_{1}$ and $\operatorname{dim}\left(T_{1}\right) \leq 3$ as in the proof of lemma 3 . If $s \geq 2, \mathbb{T}_{P}$ is the union of $s$ linear spaces, of dimensions 2 or 3 , passing through $P$ according a certain configuration $\mathcal{C}_{P} \subset \mathbb{P}^{n+4}$. By contradiction, let us assume 
that $\operatorname{dim}\left(<\mathbb{T}_{P}>\right) \geq 5$. Let $\pi_{\mathcal{L}}: \mathbb{P}^{n+4}--->\Lambda$ be any linear projection, from a suitable $(n-1)$-dimensional linear space $\mathcal{L}$ to a suitable $\Lambda \subset \mathbb{P}^{n+4}, \Lambda \simeq \mathbb{P}^{4}$, such that $\pi_{\mathcal{L}}(X)$ is isomorphic to $X$, hence $\pi_{\mathcal{L}}\left(\mathcal{C}_{P}\right)$ is isomorphic to $\mathcal{C}_{P}$. As $\operatorname{dim}\left(<\mathbb{T}_{P}>\right) \geq 5$ there is a non empty linear space $\mathcal{L}^{\prime}:=\mathcal{L} \cap<\mathbb{T}_{P}>$ such that $\pi_{\mathcal{L}}\left(\mathcal{C}_{P}\right)=\pi_{\mathcal{L}^{\prime}}\left(\mathcal{C}_{P}\right)$ where $\pi_{\mathcal{L}^{\prime}}:<\mathbb{T}_{P}>--->\Lambda$. But, as $\operatorname{dim}(\Lambda)<\operatorname{dim}\left(<\mathbb{T}_{P}>\right)$, it is not possible that $\pi_{\mathcal{L}^{\prime}}\left(\mathcal{C}_{P}\right) \simeq \mathcal{C}_{P}$, otherwise isomorphic configurations of linear spaces would have linear spans of different dimensions, so that we get a contradiction.

Lemma 5. Let $V$ and $W$ be two irreducible surfaces of $\mathbb{P}^{N}$ such that $V \cap W=$ $\langle V\rangle \cap\langle W\rangle$ is a line L. Let us assume that anyone among $V$ and $W$ is a smooth rational scroll of degree 3 in $\mathbb{P}^{4}$, or a smooth quadric in $\mathbb{P}^{3}$, or a rank 3 quadric in $\mathbb{P}^{3}$, then $\operatorname{dim}[\operatorname{Join}(V, W)]=5$ unless $V$ and $W$ are both rank 3 quadrics, having the same vertex.

Proof. Let us recall that $\operatorname{Join}(V, W):=\overline{\left\{\bigcup_{v \in V \backslash L, w \in W \backslash L}<v \cup w>\right\}} \subset \mathbb{P}^{N}$. Let $\mathcal{U} \subset \operatorname{Join}(V, W)$ be the open set $\left\{\bigcup_{v \in V \backslash L, w \in W \backslash L}<v \cup w>\right\}$, it suffices to show that $\operatorname{dim}(\mathcal{U})=5$.

Let $p$ be a generic point of $\mathcal{U}$, hence $p \in\langle v \cup w>$ for two generic points $v \in V \backslash L, w \in W \backslash L$ and we claim that, in our assumptions, $\langle v \cup w\rangle$ is the only line of $\mathcal{U}$ containing $p$. By contradiction, let us suppose that there exists another line $\left.\left\langle v^{\prime} \cup w^{\prime}\right\rangle \neq<v \cup w\right\rangle$, with $v^{\prime} \in V \backslash L, w^{\prime} \in W \backslash L$, such that $\left.p \in<v^{\prime} \cup w^{\prime}\right\rangle$. Then the two lines $\left\langle v \cup v^{\prime}\right\rangle$ and $\left\langle w \cup w^{\prime}>\right.$ intersect at a point $q \in L=$ $\langle V\rangle \cap\langle W\rangle$. But our surfaces have no trisecant lines and, for generic points $v \in V \backslash L, w \in W \backslash L$, it is not possible that $\left\langle v \cup v^{\prime}\right\rangle \cap\left\langle w \cup w^{\prime}\right\rangle$ is a point of $L$, when $\left\langle v \cup v^{\prime}>\subset V\right.$ and $\left\langle w \cup w^{\prime}>\subset W\right.$, unless $V$ and $W$ are rank 3 quadrics of common vertex $P$. In this case there are infinitely many pairs of points $v^{\prime} \in V \backslash L, w^{\prime} \in W \backslash L$ such that $\left\langle v \cup v^{\prime}\right\rangle \cap\left\langle w \cup w^{\prime}\right\rangle=P$ (and $\operatorname{dim}[\operatorname{Join}(V, W)]=4)$. So that the claim is proved. Now we can define a rational map $s: \mathcal{U}-->G(1, N)$, the Grassmannian of lines in $\mathbb{P}^{N}$, such that $s(p)=$ $<v \cup w\rangle$. Of course the generic fibre of $s$ has dimension 1 and $\operatorname{dim}(\operatorname{Im}(s))=4$, so that $\operatorname{dim}(\mathcal{U})=5$.

From theorem 1, and from the previous lemmas we get the following:

Proposition 2. Every reducible Veronese surface $X \subset \mathbb{P}^{n+4}$, according to definition 1, can be only the union $X=X_{1} \cup \ldots \cup X_{r}$ of irreducible, reduced surfaces of the following types:

- planes

- smooth quadrics of $\mathbb{P}^{3}$

- quadrics of $\mathbb{P}^{3}$ having rank 3 (quadric cones for simplicity).

Moreover only one irreducible surface of degree 2 can be contained in $X$.

Proof. From theorem 1 we know that $X=X_{1} \cup \ldots \cup X_{r}$ and that every $X_{j}$ is an irreducible, reduced, surface of minimal degree in its span. From the well known classification of irreducible, reduced surfaces of minimal degree, (see th. 0.1 of [E-G-H-P]), we have that every $X_{j}$ is a surface as above or it is a smooth Veronese surface, a smooth rational scroll of degree 4 in $\mathbb{P}^{5}$, a smooth rational scroll of degree 3 in $\mathbb{P}^{4}$.

As any surface $X_{j}$ contains a line by theorem 1, none of them can be a smooth Veronese surface. The secant variety of a smooth rational scroll of degree 4 has 
dimension 5 , so that $X$ can not contain such surfaces by condition $i i i$ ) of definition 1 .

Let us consider a smooth rational scrolls of degree 3 and let us assume, by contradiction, that it is a component of $X$, say $X_{j}$. Let $X_{k}$ be any other component of $X$, different from $X_{j}$, and suppose that $X_{k}$ is not a plane. As $X$ is a reducible Veronese surface there exists a projection $\pi_{\mathcal{L}}: \mathbb{P}^{n+4}--->\Lambda$, from a suitable linear space $\mathcal{L}$ to a suitable $\Lambda \simeq \mathbb{P}^{4}$, such that $\pi_{\mathcal{L}}(X) \simeq X$. This implies that, for any $i=1, \ldots, r, \pi_{\mathcal{L}}\left(X_{i}\right) \simeq X_{i}$, and $\pi_{\mathcal{L}}\left(X_{j}\right) \cap \pi_{\mathcal{L}}\left(X_{k}\right) \simeq X_{j} \cap X_{k}$. Recall that $\pi_{\mathcal{L}}\left(X_{j}\right) \cap$ $\pi_{\mathcal{L}}\left(X_{k}\right)$ is the intersection of two surfaces in $\mathbb{P}^{4}$ and that, by assumption, $\pi_{\mathcal{L}}\left(X_{j}\right)$ is a smooth rational scrolls of degree 3 and $\pi_{\mathcal{L}}\left(X_{k}\right)$ is another rational scrolls of degree 3 or a quadric cone or a smooth quadric. Let us examine these possibilities.

If $\pi_{\mathcal{L}}\left(X_{k}\right)$ is another rational scrolls of degree 3 then, by lemma $2, \pi_{\mathcal{L}}\left(X_{j}\right) \cap$ $\pi_{\mathcal{L}}\left(X_{k}\right)$ can not be empty, hence $\operatorname{dim}\left[\pi_{\mathcal{L}}\left(X_{j}\right) \cap \pi_{\mathcal{L}}\left(X_{k}\right)\right] \geq 0$. If $\operatorname{dim}\left[\pi_{\mathcal{L}}\left(X_{j}\right) \cap\right.$ $\left.\pi_{\mathcal{L}}\left(X_{k}\right)\right]=\operatorname{dim}\left(X_{j} \cap X_{k}\right)=0$, then $\operatorname{deg}\left[\pi_{\mathcal{L}}\left(X_{j}\right) \cap \pi_{\mathcal{L}}\left(X_{k}\right)\right]=9$ and this is not possible by corollary 2 . Hence $\operatorname{dim}\left[\pi_{\mathcal{L}}\left(X_{j}\right) \cap \pi_{\mathcal{L}}\left(X_{k}\right)\right]=\operatorname{dim}\left(X_{j} \cap X_{k}\right) \geq 1$ and, by corollary $2, X_{j} \cap X_{k}=\left\langle X_{j}\right\rangle \cap<X_{k}>$ is a line, so that $\operatorname{dim}\left[\operatorname{Join}\left(X_{j}, X_{k}\right)\right]=5$ by lemma 5 , and $\operatorname{dim}\left[\operatorname{Sec}\left(X_{j} \cup X_{k}\right)\right] \geq 5$. This implies $\operatorname{dim}[\operatorname{Sec}(X)] \geq 5$, giving a contradiction with definition 1 iii).

If $\pi_{\mathcal{L}}\left(X_{k}\right)$ is a quadric cone or a smooth quadric we can argue in the same way.

Now let us assume that $X_{k} \simeq \pi_{\mathcal{L}}\left(X_{k}\right)$ is a plane. By the above arguments, the only possibility is that the plane $\pi_{\mathcal{L}}\left(X_{k}\right)$ cuts $\pi_{\mathcal{L}}\left(X_{j}\right)$ along a line $l$, but also this case can be excluded, in fact we can consider a generic hyperplane $H$ of $\Lambda$ containing the plane $\pi_{\mathcal{L}}\left(X_{k}\right)$, the intersection $H \cap \pi_{\mathcal{L}}\left(X_{j}\right)$ is the union of $l$ and of a smooth conic $\Gamma$. As $\Gamma$ and $\pi_{\mathcal{L}}\left(X_{k}\right)$ are contained in $H \simeq \mathbb{P}^{3}$ their intersection can not be empty, so that $\operatorname{Supp}\left[\pi_{\mathcal{L}}\left(X_{j}\right) \cap \pi_{\mathcal{L}}\left(X_{k}\right)\right]$ is non contained in a line and we have a contradiction with corollary 2 .

After proving that none of the irreducible components of $X$ can be a rational scroll of degree 3 , let us exclude that $X$ has two (or more) components of degree 2 , i.e smooth quadrics or quadric cones. By contradiction, let us assume that $X$ contains two irreducible components of degree 2 , say $X_{j}$ and $X_{k}$ as before, and suppose that they are not both quadric cones with the same vertex. Then we can repeat the same argument, with the only difference that now $\left\langle X_{j}\right\rangle \simeq\left\langle X_{k}\right\rangle$ $\simeq \mathbb{P}^{3}$, and we get the same contradiction: $\operatorname{dim}[\operatorname{Sec}(X)] \geq 5$. If $X_{j}$ and $X_{k}$ are quadric cones with the same vertex $P$ we can not use lemma 5 , however in this case $T_{P}\left(X_{j}\right)=\left\langle X_{j}>\simeq \mathbb{P}^{3} \simeq<X_{k}>=T_{P}\left(X_{k}\right)\right.$ and their intersection is a line so that $\left.\operatorname{dim}\left(<\mathbb{T}_{P}\right\rangle\right) \geq 5$ and we get a contradiction with lemma 4 .

Note that, on the contrary, if $X_{j}$ is a smooth quadric or a quadric cone and $X_{k}$ is a plane we can not repeat the previous arguments to exclude the existence of quadrics in $X$.

Now we give the following:

Corollary 3. Let $X \subset \mathbb{P}^{n+4}$ be a reducible Veronese surface, according to definition 1 , for some $n \geq 1$. Then:

i) for any singular point $P \in X$ there passes only 1,2 or 3 irreducible components of $X$ and the first case occurs only when $P$ is the vertex of a quadric cone;

ii) if $P$ is a singular point of $X$, not the vertex of a quadric cone, the tangent planes at $P$ to the irreducible components of $X$ passing through $P$ (2 or 3 ) are all distinct; 
iii) if $P$ is a singular point of $X$ which it is the vertex of a quadric cone $\Gamma$ and there are at least two irreducible components of $X$ passing through $P$ :

- if the components are two, one of them is $\Gamma$ and the other one is a plane not contained in $\langle\Gamma\rangle$

- if the components are three, one of them is $\Gamma$ and the other ones are two distinct planes not contained in $\langle\Gamma\rangle$.

Proof. i) Obviously, by proposition 2, a singular point $P \in X$ belongs to only one irreducible component $X^{P}$ of $X$ if and only if $X^{P}$ is a quadric cone and $P$ is its vertex. In the other cases, let $X_{1}^{P}, \ldots, X_{s}^{P}$ be the irreducible components of $X$ containing $P, s \geq 2$. We can assume that their natural ordering is coherent with the existing ordering considered in theorem 1 . Let $T_{i}$ be the tangent space of $X_{i}^{P}$ at $P$ in $\left\langle X_{i}^{P}\right\rangle, i=1, \ldots, s$.

By lemma $3, \operatorname{dim}\left(<T_{1} \cup \ldots \cup T_{s}>\right)=\operatorname{dim}\left(<\mathbb{T}_{P}>\right) \geq \operatorname{dim}\left(T_{1}\right)+s-1 \geq s+1$. If $s \geq 4$ we would get a contradiction with lemma 4 , hence $s \leq 3$.

ii) As $P$ is not the vertex of a quadric cone, all the irreducible components of $X$ passing through $P$ are smooth at $P$ by proposition 2 and they are 2 or 3 by the previous proof. Let $T_{1}, T_{2}$ or $T_{1}, T_{2}, T_{3}$ be the tangent planes at $P$ to these components, with an ordering coherent with the ordering given by theorem 1 . By lemma $3, T_{2} \nsubseteq T_{1}$ and $T_{3} \nsubseteq<T_{1} \cup T_{2}>$ so that the planes must be distinct.

iii) By $i$ ) we have only one or two other irreducible components of $X$ passing through $P$ and they are planes by proposition 2 . The tangent space at $P$ of $\Gamma$ is $\langle\Gamma\rangle$, while the tangent spaces at $P$ of the other components concide with the components themselves, so that they can not be contained in $\langle\Gamma\rangle$, otherwise we would get a contradiction with lemma 3 for any possible ordering of these (2 or 3 ) components coherent with the ordering given by theorem 1 .

The following lemma is based on property $v$ ) of definition 1 and corollary 3 .

Lemma 6. Let $X \subset \mathbb{P}^{n+4}$ be a reducible Veronese surface, according to definition 1 , for some $n \geq 1$. Let $P$ be a singular point of $X$ such that the union $C_{P}$ of the irreducible components of $X$ passing through $P$ is a cone, i.e. (by proposition 2) the irreducible components of $X$ passing through $P$ are planes and, possibly, a quadric cone with vertex in $P$. Then if we cut $C_{P}$ with a generic hyperplane $H$, not passing through $P$, the curve $C_{P} \cap H$ is an Arithmetically Cohen-Macauley (in brief ACM) scheme.

Proof. By assumption we know that the local ring of $X$ at $P$ is a Cohen-Macaulay ring, of course it is isomorphic to the local ring of $C_{P}$ at $P$. As $C_{P}$ is a cone over $C_{P} \cap H$, with vertex $P$, the local ring of $C_{P}$ at $P$ is a Cohen-Macaulay ring if and only if $C_{P} \cap H$ is an ACM scheme.

Corollary 4. Let $X \subset \mathbb{P}^{n+4}$ be a reducible Veronese surface, according to definition 1 , for some $n \geq 1$. Let $P$ be a singular point of $X$ such that the union $C_{P}$ of the irreducible components of $X$ passing through $P$ is a cone. Then:

i) if $P$ is not the vertex of a quadric cone and there are only two components of $X$, i.e. two planes, passing through $P$, then the two planes intersect along a line;

ii) if $P$ is not the vertex of a quadric cone and there are three components of $X$, i.e. three planes, passing through $P$, then:

- the three planes intersect two by two along three lines passing through $P$, or 
- the three planes intersect along a unique line passing through $P$ and they span a 3-dimensional linear space, or

- the three planes intersect along a unique line passing through $P$ and they span a 4-dimensional linear space, or

- two planes intersect only at $P$ and the third plane cuts the other ones along two lines, passing through $P$;

iii) if $P$ is the vertex of a quadric cone and there is only another component of $X$, i.e. a plane, passing through $P$, then the plane cuts the cone only along a line of the cone.

Proof. Let us apply lemma 6 . In case $i$ ) the cone $C_{P}$ is given by two planes passing through $P$, if they intersect only at $P$ then the curve $H \cap C_{P}$ is a pair of disjoint lines in $H \simeq \mathbb{P}^{3}$ and this is not an ACM scheme.

In case $i i$ ) the cone $C_{P}$ is given by three planes passing through $P$, and the curve $H \cap C_{P}$ is a cubic curve reducible into three lines. $H \cap C_{P}$ is an ACM scheme if and only if it is: a plane cubic given by three lines in generic position or passing through a point $\left(H \simeq \mathbb{P}^{2}\right)$ or a space cubic given by a rational comb $\left(H \simeq \mathbb{P}^{3}\right)$ or three lines passing through a point and spanning a 3 -dimensional linear space $\left(H \simeq \mathbb{P}^{3}\right)$. The four possibilities give rise only to the previously described configurations.

In case $i i i)$ the cone $C_{P}$ is given by the union of a quadric cone $\Gamma$ having vertex at $P$ and a plane passing through $P$. By lemma 3 and corollary 3 iii), the plane is not contained in $\langle\Gamma\rangle$ so that it cuts $\langle\Gamma\rangle$ only at $P$ or along a line $L$ passing through $P$. If $L \in \Gamma$, then $H \cap C_{P}$ is a space cubic $\left(H \simeq \mathbb{P}^{3}\right)$ given by a smooth conic and a line cutting the conic transversally at some point, a well known ACM scheme. In the other cases $H \cap C_{P}$ would be the disjoint union of a smooth conic and a line and this is not an ACM scheme.

\section{THE MAIN RESULTS}

In this section we will get a complete classification of reducible Veronese surfaces. First of all we will prove the following theorem.

Theorem 2. Let $X \subset \mathbb{P}^{n+4}$ be a reducible Veronese surface, according to definition 1 , for some $n \geq 1$, and let us assume that all the irreducible components of $X$ are planes. Then $X=\Sigma_{n}$.

Proof. By $i i$ ) of definition 1 we have that $X$ is the union of $n+3$ planes, say $X=\Pi_{0} \cup \Pi_{1} \cup \ldots \cup \Pi_{n+2}$. By theorem 1 we can assume that the planes are ordered in such a way that, for any $j \geq 1, \Pi_{j} \cap\left(\Pi_{0} \cup \ldots \cup \Pi_{j-1}\right)$ is a line. Let us call $L_{i j}:=\Pi_{i} \cap \Pi_{j}$ when the intersection is a line and $Q_{i j}:=\Pi_{i} \cap \Pi_{j}$ when the intersection is a point. We want to use induction on $n \geq 1$.

Step one. If $n=1, X=\Pi_{0} \cup \Pi_{1} \cup \Pi_{2} \cup \Pi_{3}$ and we have to prove that $X=\Sigma_{1} \subset \mathbb{P}^{5}$. Let us consider $\Pi_{0}$ and $\Pi_{1}$, by theorem 1 they intersect along a line $L_{01}$ and $<\Pi_{0} \cup \Pi_{1}>\simeq \mathbb{P}^{3}$. Let us consider $\Pi_{2}$, by theorem 1 we know that $\Pi_{2} \cap<\Pi_{0} \cup \Pi_{1}>$ is a line $L$. By lemma $2 i i$ ) we have that $\Pi_{2} \cap \Pi_{0} \neq \emptyset$ and $\Pi_{2} \cap \Pi_{1} \neq \emptyset$, hence $L \cap \Pi_{0} \neq \emptyset$ and $L \cap \Pi_{1} \neq \emptyset$.

Let us suppose that $L$ intersects $\Pi_{0}$ only at a point $A \notin L_{01}$ and that $L$ intersects $\Pi_{1}$ only at a point $B \notin L_{01}$, so that $<\Pi_{0} \cup \Pi_{1} \cup \Pi_{2}>\simeq \mathbb{P}^{4}$. Then $A=Q_{12}$ and $B=Q_{02}$ are singular points of $X$. By corollary $4 i$ ) it is not possible that only two components of $X$ pass through $A$ and $B$, hence there is another component of $X$ passing through $A$ and there is another component of $X$ passing through $B$. As $X$ 
has only four components we have that $\Pi_{3}$ passes through $A$ and $B$, moreover, by theorem 1, $\Pi_{3} \cap\left(\Pi_{0} \cup \Pi_{1} \cup \Pi_{2}\right)$ is a line, so that $\Pi_{3} \cap\left(\Pi_{0} \cup \Pi_{1} \cup \Pi_{2}\right)=L$ and $A=Q_{13}, B=Q_{03}$. Now let us consider $A$, for instance, it is a singular point of $X$ and $\Pi_{1}, \Pi_{2}, \Pi_{3}$ pass through it, but the configuration of these planes contradicts lemma $4 \mathrm{ii}$ ), so that this case is not possible.

Let us suppose that $L=L_{01}$. In this case for any point of $L$ there pass three planes, components of $X$ (this is the maximal number by corollary $3 i$ )) intersecting among them only along the line $L$. By corollary $4 \mathrm{ii}$ ), the three planes belong to the same 3 -dimensional linear space, or generates a 4-dimensional linear space. Let us consider the last plane $\Pi_{3}$, it cuts $\Pi_{0} \cup \Pi_{1} \cup \Pi_{2}$ along a line $L^{\prime}$ by theorem 1 , hence $L^{\prime}$ belongs to $\Pi_{0}$ or to $\Pi_{1}$ or to $\Pi_{2}$ so that in any case $L^{\prime} \cap L \neq \emptyset$ and for any point in $L^{\prime} \cap L$ there pass four components of $X$, but this is a contradiction with corollary $3 i$ ).

Let us assume that $L \cap L_{01}$ is only one point $P=Q_{02}=Q_{12}$. Through $P$ there pass three planes, components of $X$ (this is the maximal number by corollary $3 i$ )), but the configuration of these planes contradicts lemma $4 \mathrm{ii}$ ), so that this case is not possible.

Therefore there is only one possibility: $L$ belongs to one of the two planes $\Pi_{0}, \Pi_{1}$ and cuts $L_{01}$ at one point $P=Q_{12}$. We can assume that $L \subset \Pi_{0}$ by reversing the role of $\Pi_{0}$ and $\Pi_{1}$, if necessary (note that we can change the position of $\Pi_{0}$ and $\Pi_{1}$ in the ordering given by theorem 1) and we have $L=L_{02}$ and $<\Pi_{0} \cup \Pi_{1} \cup \Pi_{2}>$ $\simeq \mathbb{P}^{4}$. By theorem $1, \Pi_{3} \cap<\Pi_{0} \cup \Pi_{1} \cup \Pi_{2}>$ is a line $L^{\prime}$ and, by lemma $2, L^{\prime}$ cuts every plane $\Pi_{0}, \Pi_{1}, \Pi_{2}$, hence it cuts $L$ at some point $A=Q_{03}=Q_{23}$ and it cuts $\Pi_{1}$ at some point $B=Q_{13}$. If $B \notin L_{01}$ then through $B$ would pass only two planes, components of $X$ intersecting only at $B$ and this is a contradiction with corollary $4 \mathrm{ii}$ ). Then $B \in L_{01}$ and $L^{\prime}=L_{03}$. Note that $B \neq P$ otherwise there would be four components of $X$ passing through $P$, hence the three lines: $L_{01}, L=L_{02}$, and $L^{\prime}=L_{03}$ are three lines of $\Pi_{0}$ in general position. Summing up: $\Pi_{1}, \Pi_{2}, \Pi_{3}$ cut $\Pi_{0}$ along the lines $L_{01}, L_{02}, L_{03}$, and they cut each other only at the three points $P=Q_{12}=L_{01} \cap L_{02}, B=Q_{13}=L_{01} \cap L_{03}, A=Q_{23}=L_{02} \cap L_{03}$, so that $X=\Sigma_{1}$ when $n=1$.

Step two. Let us assume that $n \geq 2$ and let us define $Y:=X \backslash \Pi_{n+2}$. We want to prove that $Y$ is a reducible Veronese surface in $\mathbb{P}^{n^{\prime}+4}$, according to definition 1 , for $n^{\prime}:=n-1 \geq 1$. Let us check properties $\left.\left.i\right), \ldots, v\right)$.

i) By theorem 1 we know that $\Pi_{n+2} \cap<\Pi_{0} \cup \ldots \cup \Pi_{n+1}>$ is a line, hence $\Pi_{n+2}$ $\cap<Y>$ is a line. As $\left.n+4=\operatorname{dim}(<X>)=\operatorname{dim}\left(<Y \cup \Pi_{n+2}\right\rangle\right)=\operatorname{dim}(<Y>$ )$+2-\operatorname{dim}\left(<Y>\cap \Pi_{n+2}\right)=\operatorname{dim}(<Y>)+1$ (we are assuming that $\left.\operatorname{dim}(\emptyset)=-1\right)$, we get that $\operatorname{dim}(\langle Y\rangle)=n+3=n^{\prime}+4$, so that $Y$ is a nondegenerate, reduced, reducible surface of pure dimension 2 in $\mathbb{P}^{n^{\prime}+4}$.

ii) $\operatorname{deg}(Y)=\operatorname{deg}(X)-1=n+2=n^{\prime}+3, \operatorname{cod}(Y)=n^{\prime}+2$.

iii) $\operatorname{dim}[\operatorname{Sec}(Y)] \leq \operatorname{dim}[\operatorname{Sec}(X)] \leq 4$

iv) $Y$ is a set of ordered planes $\Pi_{0}, \ldots, \Pi_{n+1}$ in $\mathbb{P}^{n^{\prime}+4}$ such that:

- $<\Pi_{0} \cup \ldots \cup \Pi_{n+1}>=\mathbb{P}^{n^{\prime}+4}$ by the previous check of $i$ ),

- for any $j \geq 1, \operatorname{dim}\left(\Pi_{j} \cap<\Pi_{0} \cup \ldots \cup \Pi_{j-1}>=1\right.$ by theorem 1 (recall that we have ordered all the components of $X$ according to this theorem).

Hence we can apply corollary 1 and we get that $Y$ is connected in codimension 1. 
$v$ ) To prove that $Y$ is locally Cohen-Macaulay we have to check all points of $Y$, obviously we have to check only the points of $Y \cap \Pi_{n+2}$ because for all other points of $Y$ the property follows from the fact that $X$ is locally Cohen-Macaulay.

Let $P$ be a point of $Y \cap \Pi_{n+2}$ and let us assume that there exists only one component $\Pi_{i} \subset Y$ such that $P \in \Pi_{i} \cap \Pi_{n+2}$. As $X$ is locally Cohen-Macaulay at $P$, by corollary $4 i$ ), we have that $\Pi_{i}$ intersects $\Pi_{n+2}$ along a line passing through $P$, so that when we delete $\Pi_{n+2}$ we have that $P$ is a smooth point of $Y$.

Let us assume that there are two components $\Pi_{i}, \Pi_{j} \subset Y$ such that $P \in \Pi_{i} \cap$ $\Pi_{j} \cap \Pi_{n+2}$ (two is the maximal number by corollary $\left.3 i\right)$ ). As $X$ is locally CohenMacaulay at $P$, by corollary $4 \mathrm{ii}$ ), we have the following possibilities:

- the three planes intersect two by two along three lines passing through $P$; in this case when we delete $\Pi_{n+2}$ we get that $\Pi_{i}$ intersect $\Pi_{j}$ along a line passing through $P$ and $Y$ is locally Cohen-Macaulay at $P$ (see also the proof of corollary 4 ii));

- the three planes intersect along a unique line passing through $P$ and they span a 3 -dimensional or a 4-dimensional linear space; in these cases we can argue as in the previous case and $Y$ is locally Cohen-Macaulay at $P$;

- $\Pi_{i}$ (or $\Pi_{j}$ ) and $\Pi_{n+2}$ intersect only at $P$ and the third plane cuts the other ones along two lines, passing through $P$; in this case we can argue as in the previous cases and $Y$ is locally Cohen-Macaulay at $P$;

- $\Pi_{i}$ and $\Pi_{j}$ intersect only at $P$ and $\Pi_{n+2}$ cuts the other planes along two lines, passing through $P$; in this case if we delete $\Pi_{n+2}$ we have that $Y$ is not locally Cohen-Macaulay at $P$, so we have to prove that this case is not possible; by contradiction, let us assume that the configuration of $\Pi_{i}, \Pi_{j}$ and $\Pi_{n+2}$ is as above; we can assume that $0 \leq i<j<n+2$ in the ordering given by theorem 1 , so that $\Pi_{j} \cap\left(\Pi_{0} \cup \ldots \cup \Pi_{i} \cup \ldots \cup \Pi_{j-1}\right)$ is a line $L$ passing through $P$; note that $L$ is contained in at least a plane $\Pi_{k}$ among $\Pi_{0}, \ldots, \Pi_{i}, \ldots, \Pi_{j-1}$ and that $\Pi_{k} \neq \Pi_{i}$ because $\Pi_{i} \cap \Pi_{j}=P$ (this implies $j>1$ because $\Pi_{0} \cap \Pi_{1}$ is a line), then $P \in \Pi_{k}$, so that we would have four different components of $X$ passing through $P$ and we would have a contradiction with corollary $3 i$ ).

Step three. Now let us proceed by induction on $n \geq 1$. If $n=1$ theorem 2 is true by step one. Let us assume that the theorem is true for any $X$ in $\mathbb{P}^{5}, \mathbb{P}^{6}, \ldots, \mathbb{P}^{n+3}$ and let us prove the theorem for $X \subset \mathbb{P}^{n+4}$. As in step two we can decompose $X=Y \cup \Pi_{n+2}$ and we know that $Y$ is a reducible Veronese surface in $\mathbb{P}^{n+3}$ according to definition 1 , by step two. By induction we can say that $Y=\Sigma_{n-1}$ so that $X=\Sigma_{n-1} \cup \Pi_{n+2}$. By theorem 1 we have that $\Sigma_{n-1} \cap \Pi_{n+2}$ is a line $L$ and, as above, $L$ is contained in at least a plane among $\Pi_{0}, \ldots, \Pi_{n+1}$.

By contradiction, let us assume that $L \subset \Pi_{i}$ for some $i>0$ and let us consider the line $L_{0 i} . L$ can not contain any point $Q_{i j} \in L_{0 i}(j=1, \ldots, n+1, j \neq i)$ and a fortiori $L \neq L_{0 i}$ otherwise we would have four different components of $X$ passing through $Q_{i j}: \Pi_{0}, \Pi_{i}, \Pi_{j}, \Pi_{n+2}$, a contradiction with corollary $3 i$ ). So that $L \cap L_{0 i}$ is a point $P \neq Q_{i j}$ for any $j=1, \ldots, n+1, j \neq i$, and the point $P \in X$ belongs exactly to $\Pi_{n+2}, \Pi_{i}, \Pi_{0}$, but this configuration contradicts corollary 4 ii) because $\Pi_{n+2} \cap \Pi_{i}=L, \Pi_{n+2} \cap \Pi_{0}=P, \Pi_{i} \cap \Pi_{0}=L_{0 i}$ and $L \cap L_{0 i}=P$.

Therefore $L \subset \Pi_{0}$ (i.e. $L=L_{0(n+2)}$ ) and to prove that $X=\Sigma_{n}$ we have only to show that the lines $L_{0 i}$ with $i=1, \ldots, n+1$ and $L$ are in general position on $\Pi_{0}$ i.e. that the curve given by their union has no triple points. But this curve has a triple 
point if and only if $L$ passes through some point $Q_{i j}$ for some $i, j=1, \ldots, n+1$ $i \neq j$, (recall that $Y=\Sigma_{n-1}$ ) and we have proved that this is not possible.

To classify reducible Veronese surfaces containing a quadric we need other lemmas.

Lemma 7. Let $V=V_{1} \cup \ldots \cup V_{r} \subset \mathbb{P}^{N}$ be a non degenerate, reducible, reduced, surface of pure dimension 2 , whose irreducible components are $V_{1}, \ldots, V_{r}$. Let $W \subset V$ be a proper subvariety of $V$ such that $W=V_{1} \cup \ldots \cup V_{\rho}$ with $1 \leq \rho<r$. Assume that $V$ and $W$ are connected in codimension 1 . Then there exists at least a component $V_{i} \subset V$ with $\rho<i \leq r$ such that $\operatorname{dim}\left(W \cap V_{i}\right)=1$ and $W \cup V_{i}$ is connected in codimension 1 .

Proof. If $\operatorname{dim}\left(W \cap V_{i}\right) \leq 0$ for any irreducible component $V_{i} \subset V$ with $\rho<i \leq r$, then $\operatorname{dim}\left[W \cap\left(V_{\rho+1} \cup \ldots \cup V_{r}\right)\right] \leq 0$, but this is not possible, otherwise $V \backslash[W \cap$ $\left.\left(V_{\rho+1} \cup \ldots \cup V_{r}\right)\right]$ would be not connected while we are assuming that $V$ is connected in codimension 1 . Hence, by changing the ordering of $V_{\rho+1}, \ldots, V_{r}$ if necessary, we can assume that $\operatorname{dim}\left(W \cap V_{\rho+1}\right) \geq 1$. It is not possible that $\operatorname{dim}\left(W \cap V_{\rho+1}\right) \geq 2$, otherwise the irreducible surface $V_{\rho+1}$ would be a component of $W$, so that $\operatorname{dim}\left(W \cap V_{\rho+1}\right)=1$.

Now let us consider $W \cup V_{\rho+1} . W$ is connected in codimension 1 by assumptions, $V_{\rho+1}$ is connected in codimension 1 because it is an irreducible surface; as $\operatorname{dim}(W \cap$ $\left.V_{\rho+1}\right)=1$ we have that $W \cup V_{\rho+1}$ is connected in codimension 1 too.

Lemma 8. Let $X \subset \mathbb{P}^{n+4}$ be a reducible Veronese surface, according to definition 1 , for some $n \geq 1$, and let $X_{1}, \ldots, X_{r}$ be its irreducible components. Let us assume that $X$ contains a quadric $Q$. Then:

i) $r=n+2$;

ii) there exists an ordering $X_{1}, \ldots, X_{n+2}$ according to theorem 1 such that $Q=X_{1}$.

Proof. $i$ ) Recall that, by proposition $2, Q$ is the only component of $X$ having degree $\geq 2$, so that $n+3=\operatorname{deg}(X)=2+r-1$, hence $r=n+2$.

ii) Let us put $X_{1}=Q$. By lemma 7 there is (at least) another component $X_{\bar{i}} \subset$ $X$ such that $\operatorname{dim}\left(Q \cap X_{\bar{i}}\right)=1$ and $Q \cup X_{\bar{i}}$ is connected in codimension 1, moreover $X_{\bar{i}}$ is a plane. By corollary $2 Q \cap X_{\bar{i}}$ is a line. If we put $X_{2}=X_{\bar{i}}$ we have that $X_{1} \cap X_{2}=\left\langle X_{1}\right\rangle \cap\left\langle X_{2}\right\rangle$ and the intersection is a line.

As $n \geq 1$ we have $r \geq 3$, so that there exists at least another component. Now let us apply lemma 7 to $X_{1} \cup X_{2}$, which is connected in codimension 1 , and there is (at least) another component $X_{\bar{i}} \subset X$ such that $\operatorname{dim}\left[\left(X_{1} \cup X_{2}\right) \cap X_{\bar{i}}\right]=1$ and $X_{1} \cup X_{2} \cup X_{\bar{i}}$ is connected in codimension 1 , moreover $X_{\bar{i}}$ is a plane, and so on. By applying lemma 7 a suitable number of times we get an ordering $X_{1}, \ldots, X_{n+2}$ such that $X_{1}=Q$ and, for any $j \geq 2, \operatorname{dim}\left[X_{j} \cap\left(X_{1}, \ldots, X_{j-1}\right)\right]=1$ and $X_{1} \cup \ldots \cup X_{j}$ is connected in codimension 1 .

Let us consider $\left\langle X_{j}\right\rangle \cap\left\langle X_{1} \cup \ldots \cup X_{j-1}\right\rangle=X_{j} \cap\left\langle X_{1} \cup \ldots \cup X_{j-1}\right\rangle$ for any $j \geq 2$ and we have $\operatorname{dim}\left(X_{j} \cap<X_{1} \cup \ldots \cup X_{j-1}>\right) \geq \operatorname{dim}\left[X_{j} \cap\left(X_{1} \cup \ldots \cup X_{j-1}\right)\right]=1$. Let us put $a_{j}:=\operatorname{dim}\left(X_{j} \cap<X_{1} \cup \ldots \cup X_{j-1}>\right)$ for any $j \geq 3$, so that:

$\operatorname{dim}\left(<X_{1} \cup X_{2}>\right)=4$

$\operatorname{dim}\left(<X_{1} \cup X_{2} \cup X_{3}>\right)=\operatorname{dim}\left(<<X_{1} \cup X_{2}>\cup X_{3}>\right)=$ $=\operatorname{dim}\left(<X_{1} \cup X_{2}>\right)+2-a_{3}$

$\operatorname{dim}\left(<X_{1} \cup X_{2} \cup X_{3} \cup X_{4}>\right)=\operatorname{dim}\left(<<X_{1} \cup X_{2} \cup X_{3}>\cup X_{4}>\right)=$

$=\operatorname{dim}\left(<X_{1} \cup X_{2}>\right)+2-a_{3}+2-a_{4}$ 
$\operatorname{dim}\left(<X_{1} \cup X_{2} \cup \ldots \cup X_{n+2}>\right)=\operatorname{dim}\left(<<X_{1} \cup X_{2} \cup \ldots \cup X_{n+1}>\cup X_{n+2}>\right)=$ $=\operatorname{dim}\left(<X_{1} \cup X_{2}>\right)+2-a_{3}+2-a_{4}+\ldots \ldots+2-a_{n+2}=$ $=4+2 n-\sum_{j=3}^{n+2} a_{j}=n+4$.

Hence $\sum_{j=3}^{n+2} a_{j}=n$. As $a_{j} \geq 1$ for any $j \geq 3$ we have in fact $a_{j}=1$ for any $j \geq 3$, so that $1=\operatorname{dim}\left(X_{j} \cap<X_{1} \cup \ldots \cup X_{j-1}>\right)=\operatorname{dim}\left[X_{j} \cap\left(X_{1} \cup \ldots \cup X_{j-1}\right)\right]$ for any $j \geq 2$ (the case $j=2$ was considered previously) and $X_{j} \cap<X_{1} \cup \ldots \cup X_{j-1}>$ is obviously a line.

To prove lemma $8 \mathrm{ii}$ ) now we have to show that $X_{j} \cap<X_{1} \cup \ldots \cup X_{j-1}>$ $=X_{j} \cap\left(X_{1} \cup \ldots \cup X_{j-1}\right)$ for any $j \geq 2$. As above, the case $j=2$ was considered previously, so we can assume $j \geq 3$ and recall that $X_{j}$ is a plane. As $X_{j} \cap<$ $X_{1} \cup \ldots \cup X_{j-1}>\supseteq X_{j} \cap\left(X_{1} \cup \ldots \cup X_{j-1}\right)$ and $X_{j} \cap<X_{1} \cup \ldots \cup X_{j-1}>$ is a line we have only to show that $X_{j} \cap\left(X_{1} \cup \ldots \cup X_{j-1}\right)$ is a line. As $\operatorname{dim}\left[X_{j} \cap\left(X_{1} \cup \ldots \cup\right.\right.$ $\left.\left.X_{j-1}\right)\right]=1$ there exists at least one component $X_{i}$, with $1 \leq i \leq j-1$, such that $\left.\operatorname{dim}\left[X_{j} \cap X_{i}\right)\right]=1$, hence $X_{j} \cap X_{i}$ is a line $L_{i j}$ by corollary 2. Moreover there are no other points $P \in X_{j} \cap\left(X_{1} \cup \ldots \cup X_{j-1}\right), P \notin L_{i j}$, otherwise $X_{j}$ would be contained in $<X_{1} \cup \ldots \cup X_{j-1}>$ and this is not possible as $\operatorname{dim}\left(X_{j} \cap<X_{1} \cup \ldots \cup X_{j-1}>\right)=1$. It follows that, for any $j \geq 3, X_{j} \cap\left(X_{1} \cup \ldots \cup X_{j-1}\right)$ is a line and we are done.

Now we can conclude this section with the following theorems.

Theorem 3. Let $X \subset \mathbb{P}^{n+4}$ be a reducible Veronese surface, according to definition 1 , for some $n \geq 1$, and let $X_{1}, \ldots, X_{r}$ be its irreducible components. Let us assume that $X$ contains a smooth quadric $Q$. Then $n=1, r=3, X=Q \cup X_{1} \cup X_{2}$, where $X_{1}$ and $X_{2}$ are planes, and we have only two possibilities:

a) $Q, X_{1}, X_{2}$ intersect transversally along a unique line $L=Q \cap X_{1} \cap X_{2}$;

b) $X_{1}$ and $X_{2}$ cut $Q$ along two lines intersecting at a point $P=X_{1} \cap X_{2}$.

Proof. By lemma 8 we know that $r=n+2 \geq 3$ and there exists an ordering $X_{1}, \ldots, X_{n+2}$ given by theorem 1 such that $X_{1}=Q, X_{i}$ is a plane for any $i \geq 2$ and $X_{2}$ cuts $Q$ along a line $L$. Now let us consider the plane $X_{3}$ cutting $Q \cup X_{2}$ and $<Q \cup X_{2}>\simeq \mathbb{P}^{4}$ along a line $L^{\prime}$ by theorem 1 . We have some cases to consider.

1) Let us assume that $L^{\prime} \subset X_{2}$ and $L^{\prime} \neq L$ so that $L^{\prime} \cap L$ is a point $\bar{P} \in Q$, then $\left\langle X_{2} \cup X_{3}>\simeq \mathbb{P}^{3}, L=\left\langle X_{2} \cup X_{3}>\cap\langle Q\rangle,\left\langle Q \cup X_{2} \cup X_{3}>\simeq \mathbb{P}^{5}\right.\right.\right.$. and $\bar{P}=Q \cap X_{3}$ so that $\left\langle Q \cup X_{3}>=<Q \cup X_{2} \cup X_{3}>\simeq \mathbb{P}^{5}\right.$. This case is not possible, in fact, let $P$ be a generic point in $\left\langle Q \cup X_{3}>\right.$; note that, in particular, this means that $P \notin<Q>\cup X_{3}$ and $P \notin<T_{\bar{P}}(Q) \cup X_{3}>\simeq \mathbb{P}^{4}$. Let us consider the 3-dimensional linear space $\Lambda_{P}:=<P \cup X_{3}>\subset<Q \cup X_{3}>\simeq \mathbb{P}^{5}$. We have that $\Lambda_{P} \cap<Q>$ is a line $L_{P}$ passing through $\bar{P}$ and that there exists (at least) another point $P^{\prime} \in Q$ on $L_{P}$ with $\bar{P} \neq P^{\prime}$; recall that $P \notin<T_{\bar{P}}(Q) \cup X_{3}>$ so that the line $L_{P}$ is not tangent to $Q$. Now the line $P P^{\prime} \in \Lambda_{P}$ cuts $X_{3}$ at some point $P^{\prime \prime} \neq \bar{P}$ (otherwise $L_{P}=P P^{\prime}$ and $P \in<Q>$ ) so that $P \in \operatorname{Sec}\left(Q \cup X_{3}\right) \subset \operatorname{Sec}(X)$. It follows that the generic point of $<Q \cup X_{2} \cup X_{3}>\simeq \mathbb{P}^{5}$ is contained in $\operatorname{Sec}(X)$, hence $\operatorname{dim}[\operatorname{Sec}(X)] \geq 5$ and we get a contradiction with $i i i)$ of definition 1 .

2) Let us assume that $L^{\prime} \subset X_{2}$ and $L^{\prime}=L$. By contradiction let us assume that there exists another plane $X_{4}$ in $X$. Then $X_{4} \cap\left(Q \cup X_{2} \cup X_{3}\right)$ is a line $L^{\prime \prime}$, but $L^{\prime \prime}$ can not be contained in $X_{2}$ or in $X_{3}$ otherwise we would have four components of $X$ passing through a point and this is not possible by corollary $3 i$ ), hence $L^{\prime \prime} \subset Q$. 
Analogously we have $L^{\prime \prime} \cap L=\emptyset$, but in this case $X_{4}$ must intersect $X_{2}$ at some point $P$ by lemma $2 \mathrm{ii}$ ), so that $X_{4}=\left\langle P \cup L^{\prime \prime}\right\rangle$ would be contained in < $Q \cup X_{2} \cup X_{3}>$ and this is not possible by lemma $8 \mathrm{ii}$ ). Hence there are only two planes in $X$ and we get $a$ ).

3) Let us assume that $L^{\prime} \subset Q$ and that $L \cap L^{\prime}=\emptyset$. Then $X_{3} \cap X_{2}$ would be a point $P$ by lemma $2 \mathrm{ii}$ ) and we would get a contradiction by arguing as above: $X_{3}=\left\langle L^{\prime} \cup P\right\rangle$ would be contained in $\left\langle Q \cup X_{2}\right\rangle$.

4) Let us assume that $L^{\prime} \subset Q$ and that $L \cap L^{\prime}$ is a point $P$ and, by contradiction, let us assume that there exists another plane $X_{4}$ in $X$. Then $X_{4} \cap\left(Q \cup X_{2} \cup X_{3}\right)$ is a line $L^{\prime \prime}$. If $L^{\prime \prime} \subset Q, L^{\prime \prime} \neq L, L^{\prime \prime} \neq L^{\prime}$ then $X_{4} \cap X_{2}=\emptyset$ or $X_{4} \cap X_{3}=\emptyset$ and this is not possible by lemma $2 \mathrm{ii}$ ), on the other hand if $L^{\prime \prime}=L$ or $L^{\prime \prime}=L^{\prime}$ we would have four components of $X$ passing through a point and this is not possible by corollary $3 i$ ). So that $L^{\prime \prime} \nsubseteq Q$ and $L^{\prime \prime} \subset X_{2}$ or $L^{\prime \prime} \subset X_{3}$. Now let us suppose that $L^{\prime \prime} \subset X_{2}$ (the other case is similair), if $P \notin L^{\prime \prime}$ then $X_{4} \cap X_{3}=\emptyset$ and this is not possible by lemma $2 \mathrm{ii}$ ), on the other hand if $P \in L^{\prime \prime}$ we would have four components of $X$ passing through a point and this is not possible by corollary $3 i$ ). Hence there are only two planes in $X$ and we get $b$ ).

To complete the proof of theorem 3 now we have to prove that the surfaces $X$ in cases $a$ ) and $b$ ) are reducible Veronese surfaces according to definition 1: $i$ ), $i i$ ) and $i v$ ) are obvious; for $i i i)$ let us remark that $\operatorname{Sec}(X)$ is the union of a finite number of linear spaces of dimension $\leq 4$; for $v$ ) we can apply lemma 1 .

Theorem 4. Let $X \subset \mathbb{P}^{n+4}$ be a reducible Veronese surface, according to definition 1 , for some $n \geq 1$, and let $X_{1}, \ldots, X_{r}$ be its irreducible components. Then none of the components of $X$ can be a quadric cone.

Proof. By contradiction, let us suppose that $X$ contains a quadric cone $\Gamma$ of vertex $P_{\Gamma}$. By lemma 8 we know that $r=n+2 \geq 3$ and there exists an ordering $X_{1}, \ldots, X_{n+2}$ such that $\Gamma=X_{1}$, the other components are planes and $X_{2} \cap \Gamma$ is a line $L$ passing through $P_{\Gamma}$. Let us consider the plane $X_{3}$ ant let us remark that $P_{\Gamma} \notin X_{3}$, in fact the union of the tangent spaces to $\Gamma$ and $X_{2}$ at $P_{\Gamma}$ spans the 4-dimensional linear space $<\Gamma \cup X_{2}>$ and $X_{3} \notin<\Gamma \cup X_{2}>$ by theorem 1 , so that, if $P_{\Gamma} \in X_{3}$, we would get a contradiction with lemma 4 for $P=P_{\Gamma}$.

On the other hand we know that $X_{3} \cap\left(\Gamma \cup X_{2}\right)$ is a line $L^{\prime}$ by theorem 1 . As $P_{\Gamma} \notin X_{3}$ we have that $L^{\prime} \nsubseteq \Gamma$, so that $L^{\prime} \subset X_{2}$ and it cuts $\Gamma$ only at a point $\bar{P} \in L$, $\bar{P} \neq P_{\Gamma}$. Hence $X_{3}$ and $\Gamma$ are in the same configuration as $X_{3}$ and $Q$ in case 1) of theorem 3 , so that we can argue as above and we can prove that this case is not possible. Therefore $X_{3}$ does not exist and we get a contradiction as $r \geq 3$.

Remark 3. The above theorems 2, 3 and 4, taking into account proposition 2, give a complete classification of the reducible Veronese surfaces according to definition 1. It follows that the generic surfaces $S_{n}$, embedded in $\mathbb{P}^{n+4}$, introduced by Floystad in $[\mathrm{F}]$, are in fact surfaces $\Sigma_{n}$ for any $n \geq 2$. If $n=2$ the proof was made in §2. If $n \geq 3$ we have only to check that any generic $S_{n}$ satifies definition 1: in $[\mathrm{F}]$ it is proved that $S_{n}$ is non degenerated and that iii) and $v$ ) hold; from $v$ ) it follows that $S_{n}$ is reduced, of pure dimension 2, and that iv) holds (see Remark 1); ii) follows from direct calculation as in §2; to have i) it suffices to show that $S_{n}$ is reducible, if not, from the classification of irreducible, reduced surfaces of minimal degree (see the beginning of the proof of proposition 2) it would follow $\operatorname{deg}\left(S_{n}\right) \leq 4$, while $\operatorname{deg}\left(S_{n}\right) \geq 6$ as $n \geq 3$. 
Remark 4. Reducible Veronese surface $X$ are not locally complete intersections. In fact let us consider any triple point $P \in X$ and let $Y_{p}$ be any generic hyperplane section of $X$ passing through $P$. If $X$ is locally complete intersection at $P$ then $Y_{p}$ is locally complete intersection at $P$ too (see for instance [B-H] Th. 2.3.4). If $X=\Sigma_{n}$ then $Y_{p}$ is the union of 3 lines passing through $P$, spanning a 3-dimensional linear space. If $X$ is one of the cases a), $b$ ) of theorem 3 then $Y_{P}$ is the union of a smooth conic and two lines passing through $P$, spanning a 4-dimensional linear space. In any case $Y_{P}$ is not locally complete intersection at $P$.

Remark 5. Reducible Veronese surfaces are not even locally Gorenstein. Let X, $P, Y_{P}$ be as in remark 4. If $X$ is locally Gorenstein at $P$ then the dualizing sheaf $\omega_{X}$ is free at $P$ and it has rank 1 (see [E] p.532). By adjunction we have that $\omega_{Y_{P}}=\left(\omega_{X}+H\right)_{\mid Y_{P}}$ where $H$ is the Cartier divisor of $X$ corresponding to $Y_{P}$ (see lemma 1.7.6 of $[\mathrm{B}-\mathrm{S}]$ ), so that $\omega_{Y_{P}}$ is free at $P$ and it has rank 1 too. But this is not possible: let $f: \overline{Y_{P}} \rightarrow Y_{P}$ be the normalization of $Y_{P}$, note that $f$ is a triple unramified covering locally at $P$. The conductor sheaf $\mathcal{C}$ of $\mathcal{O}_{\overline{Y_{P}}, P}$ in $\mathcal{O}_{Y_{P}, P}$ is the maximal ideal of $\mathcal{O}_{Y_{P}, P}$, hence $\operatorname{dim}_{\mathbb{C}}\left(\mathcal{O}_{Y_{P}, P} / \mathcal{C}\right)=1$, on the other hand $\operatorname{dim}_{\mathbb{C}}\left(\mathcal{O}_{\overline{Y_{P}}, P} / \mathcal{O}_{Y_{P}, P}\right)=2$ and this is a contradiction because $\operatorname{dim}_{\mathbb{C}}\left(\mathcal{O}_{\overline{Y_{P}}, P} / \mathcal{O}_{Y_{P}, P}\right)=$ $\operatorname{dim}_{\mathbb{C}}\left(\mathcal{O}_{\overline{Y_{P}}, P} / \mathcal{C}\right)+\operatorname{dim}_{\mathbb{C}}\left(\mathcal{O}_{Y_{P}, P} / \mathcal{C}\right)=2+1=3$.

\section{REFERENCES}

[B-S] M.C.Beltrametti-A.J.Sommese: "The adjunction theory of complex projective varieties" Walter de Gruyter, Berlin, 1995.

[B-H] W.Bruns-J.Herzog: "Cohen-Macaulay rings" Cambridge studies in advanced math. 39, Cambridge Univ. Press 1993.

[E] D.Eisenbud: "Commutative Algebra with a View Towards Algebraic Geometry" G.T.M. 150, Springer, New York, 1995.

[F] G.Floystad: "Monads on projective spaces" Comm. Algebra 28 (12) (2000), p. 55035516.

[E-G-H-P] D.Eisenbud-M.Green-K.Hulek-S.Popescu: "Small schemes and varieties of minimal degree" Amer. J. Math. 128 (6) (2006), p. 1345-1389.

[H] R.Hartshorne: "Complete intersections and connectedness" Amer. J. Math. 84 (1962), p.497-508.

[X] S.Xambò: "On projective varieties of minimal degree" Collectanea Math. 32 (2) (1981), p.149-163.

Dipartimento di Matematica Univ. di Milano, via C. Saldini 50 20133-Milano (Italy)

E-mail address: alzati@mat.unimi.it

Dipartimento di Matematica Univ. di Trento, via Sommarive 14 38050-Povo (TN) (ITALY)

E-mail address: ballico@science.unitn.it 